\title{
Latent variable and clustering methods in intersectionality research: systematic review of methods applications
}

\author{
Greta R. Bauer $^{1}$ (1) $\cdot$ Mayuri Mahendran ${ }^{1} \cdot$ Chantel Walwyn $^{1} \cdot$ Mostafa Shokoohi $^{2}$ (])
}

Received: 11 June 2021 / Accepted: 31 October 2021 / Published online: 13 November 2021

(c) The Author(s) 2021

\begin{abstract}
Purpose An intersectionality framework has been increasingly incorporated into quantitative study of health inequity, to incorporate social power in meaningful ways. Researchers have identified "person-centered" methods that cluster withinindividual characteristics as appropriate to intersectionality. We aimed to review their use and match with theory.

Methods We conducted a multidisciplinary systematic review of English-language quantitative studies wherein authors explicitly stated an intersectional approach, and used clustering methods. We extracted study characteristics and applications of intersectionality.

Results 782 studies with quantitative applications of intersectionality were identified, of which 16 were eligible: eight using latent class analysis, two latent profile analysis, and six clustering methods. Papers used cross-sectional data (100.0\%) primarily had U.S. lead authors $(68.8 \%)$ and were published within psychology, social sciences, and health journals. While $87.5 \%$ of papers defined intersectionality and $93.8 \%$ cited foundational authors, engagement with intersectionality method literature was more limited. Clustering variables were based on social identities/positions (e.g., gender), dimensions of identity (e.g., race centrality), or processes (e.g., stigma). Results most commonly included four classes/clusters (60.0\%), which were frequently used in additional analyses. These described sociodemographic differences across classes/clusters, or used classes/clusters as an exposure variable to predict outcomes in regression analysis, structural equation modeling, mediation, or survival analysis. Author rationales for method choice included both theoretical/intersectional and statistical arguments. Conclusion Latent variable and clustering methods were used in varied ways in intersectional approaches, and reflected differing matches between theory and methods. We highlight situations in which these methods may be advantageous, and missed opportunities for additional uses.
\end{abstract}

Keywords Systematic review $\cdot$ Intersectionality $\cdot$ Health equity $\cdot$ Research methods $\cdot$ Latent variable methods $\cdot$ Clustering methods

\section{Introduction}

As a framework for incorporating social power, heterogeneity, and community relevance into health research, intersectionality has gained prominence as an analytic approach to qualitative and more recently quantitative methods [1].

Greta R. Bauer

gbauer@uwo.ca

1 Epidemiology and Biostatistics, Schulich School of Medicine and Dentistry, Western University, London, ON, Canada

2 Social and Behavioural Health Sciences, Dalla Lana School of Public Health, University of Toronto, Toronto, ON, Canada
Emerging from U.S. Black feminist communities $[2,3]$ and into Black feminist academic work in legal studies and sociology [4-6], intersectionality focuses on social power structures and the ways oppression is configured at intersections of race, gender, class, and other axes of marginalization [6]. Importantly, it centers the experiences of those at the social margins in an embodied way [7, 8], with the central recognition that experiences and well-being are shaped by mutually constituted social identities or positions. This is to say that, for example, the experiences of Black lesbians cannot be understood by adding together the average effects of being Black, being a sexual minority, and being a woman [7]. As such, as intersectionality has been taken up within quantitative research, researchers have sought out methods that allow variables to act together in ways that may better reflect 
intersectional analytic approaches. One such set of methods involves grouping individuals based on how multiple variables cluster together, producing new categories reflecting meaningful patterns of multiple attributes. Such methods fall into the broad categories of latent variable methods and clustering methods.

Health researchers have highlighted latent variable methods such as latent class analysis (LCA) and latent profile analysis (LPA) as data-driven analytical approaches for examining intersectional stigma [9]—a concept that represents the influence of combined and overlapping oppressions to form a distinct positionality [10]. These analytic methods take a "person-centered" approach by which subgroups of individuals are identified based on their stigma experiences, rather than a "variable-centered" approach in which individuals are not grouped and stigma is assumed to impact everyone similarly [11]. Latent variable methods are model-based approaches; they assume an underlying statistical model for the population from which the data were obtained and identify unobservable (latent) groups within that population [12]. LCA uses a set of observed categorical variables to identify latent classes [13], wherein individuals are homogenous within classes (i.e., with high probability of similar response patterns regarding the measured variables), while heterogeneous across classes [11]. LCA assumes conditional independence, that is, the measured variables are conditionally independent given latent class membership [13]. Given the type of observed data, multiple extensions exist, including LPA which similarly creates classes/profiles but using continuous variables, and latent transition models (LTA) used for longitudinal data [14]. As model-based methods, goodness-of-fit statistics such as the Akaike information criterion [15], Bayesian information criterion [16], sample size adjusted BIC [17], and entropy [18] are available to aid in decision-making about the number and features of the latent groups. Fit is one consideration in addition to parsimony, model stability, and interpretability [13]. Details regarding LCA model building, selection, interpretation, and presentation of results have been described elsewhere [19, 20].

The second broad class of methods includes non-modelbased clustering methods such as hierarchical cluster analysis (HCA) and non-hierarchical cluster analysis (e.g., $\mathrm{K}$-means clustering) [21]. Like latent variable methods, they allow for classification based on clustering on a range of measured variables. Unlike latent variable models that intend to recover unobservable observations in the data (using multiple observed variables), their main goal is only data-driven identification of clusters, and goodness-of-fit statistics are not available [20, 22]. Agglomerative HCA, for example, considers each individual as a single cluster; then, most-similar clusters are grouped together in an iterative process until bigger clusters, all mutually exclusive, are created $[21,22]$. The similarities or dissimilarities of the identified clusters are taken into account using distance measures (e.g., Euclidean distance algorithm) or similarity measures, followed by cluster validation [22]. Also, multiple cluster agglomeration (or linkage) methods have been proposed for measuring dissimilarities between two clusters, for example, nearest neighbor, centroid clustering, and Ward's method [22]. Unlike latent variable methods, clustering methods typically accommodate multiple variable types within one analysis, as values are transformed using standardization techniques. Clustering results are typically depicted in a tree-like dendrogram.

The relationship between intersectionality and latent variable or clustering methods requires elucidation. Core tenets of intersectionality suggest different potential uses for such person-centered approaches. For example, the most foundational tenet that social categories are not independent, but interlocking [4, 23, 24] may suggest creation of clusters/ classes using social identities or positions such as gender, ethnoracial group, and education. Another tenet is that these identities/positions are linked to social and structure inequity [23], which may suggest creation of clusters/classes related to social-structural processes such as sexism, racism, or classism. However, similar approaches based in other frameworks (e.g., social determinants of health) have been used without labeling them intersectional [25]. In addition, generation of new categorical measures produced using these types of methods may be driven by pragmatic rather than theoretical objectives, for example to reduce variable numbers. Moreover, classes or clusters are often not themselves the ultimate goal of research studies. Rather, these new categorical variables are used within additional analyses in varying ways. Ultimate goals may be descriptive or analytic, either describing inequalities in health or other outcomes across classes/clusters, or using these new classifications in analytic studies of processes that drive inequalities [26].

In this review, we sought to evaluate uses of latent variable and clustering methods across disciplines, in academic articles that explicitly stated an intersectional approach. We aimed to describe study characteristics, engagement with intersectionality, and methods used, and to evaluate match between intersectionality and methods to provide guidance for future health equity research.

\section{Methods}

\section{Search strategy}

The systematic review was conducted according to the Preferred Reporting Items for Systematic Reviews and MetaAnalyses (PRISMA) guidelines [27], though the standard assessment of the risk of bias in included studies was not deemed relevant as our interest was characterizing methods 
rather than evaluating evidence from study results. As a part of a larger intersectionality methods review [1], researchers conducted a systematic search of all quantitative Englishlanguage research articles from 1989 (the year the word was introduced in academic literature [5]), through May 12, 2020, wherein the authors explicitly stated they were applying an intersectionality theoretical framework. A search of Scopus (including Medline) and ProQuest Political Science and Public Administration (including PsycINFO) identified papers with titles, abstracts or keywords containing "intersectional*". Titles and keywords containing "qualitative" were excluded from the search [1].

\section{Selection strategy}

Papers were de-duplicated using Covidence Systematic Review Software [28]. To determine eligibility, papers were jointly title and abstract screened by two independent reviewers, and full-text articles were screened by a single reviewer. Interrater reliability during screening was $92.5 \%$, and conflicting decisions were resolved via consensus-based discussions. Papers were deemed eligible for inclusion into the study if they were peer-reviewed original quantitative or mixed methods studies or methods papers that explicitly stated the use of an intersectional framework. Measure development or validation papers were excluded, as while clustering methods play a role in scale validation it is for a very specific purpose that is not usually intersectional in nature. Finally, all papers that did not use latent variable or clustering analyses in an explicitly intersectional way were excluded. In total, 16 papers were identified as eligible for inclusion.

\section{Data extraction}

Data items were extracted into an Excel sheet, which had been pilot tested and revised to improve clarity. Data on latent variable or cluster methods were extracted by two independent reviewers ( $\mathrm{MM}$ and $\mathrm{CW}$ ), with data checks conducted to validate extraction results. The following data items were extracted: (a) Article characteristics (journal discipline, country of first author's home institution, research approach, study design, sampling method, sample size, use of a health outcome), (b) Incorporation of the intersectionality framework (explicitly defining intersectionality, citing foundational authors, citing key methodological papers), (c) Latent variable and clustering methods (type of method used, rationale for method used, variables used in method, number of groups created, approach used to choose final number of groups, group variable names), and (d) Use of group variable in additional analyses (type of statistical method conducted using the group variable, role of group variable in analysis, ability for the results to vary by intersection, reporting results for all intersections). Journal discipline was categorized by grouping initial classifications from within Ulrich's Serials Analysis System [1, 29]. Citation of foundational intersectional authors was recorded as any citation of the Combahee River Collective [2], legal scholar Kimberlé Crenshaw [4, 5], or sociologist Patricia Hill Collins [6]. Number of key methodological intersectionality papers cited was coded based on 45 papers identified in our earlier large interdisciplinary systematic review of quantitative intersectional methods in papers published from 1989 to 2020 [1]. Finally, quotes were extracted wherein authors discussed their rationale for their methods choices.

\section{Data analysis}

Measures of frequency were estimated for each extracted data item using SAS version 9.4.1. [30]. Extracted author quotes were reviewed to identify elements of authors' rationales for their latent variable or clustering methods use.

\section{Results}

Following full-text review, 782 quantitative intersectionality application papers remained, of which $16(2.0 \%)$ used a latent variable or clustering method and were included in extraction and analysis for this paper (Fig. 1). Table 1 summarizes the characteristics of included papers and indicators of their engagement with intersectionality. Of the 16 papers, $10(62.5 \%)$ included a health-related outcome, while some others focused on areas such as education that may be considered social determinants of health. Papers were primarily in psychology, social sciences, and health disciplines, had U.S. lead authors, were quantitative only and cross-sectional, and traversed the full range of sample sizes. Fourteen of 16 papers (87.5\%) defined intersectionality, and 15 (93.8\%) cited foundational authors. Engagement with methodology papers varied, with 4 (25.0\%) citing none of the 45 methods papers and 7 (43.8\%) citing just one.

Details on applications of intersectionality in these papers are summarized in Table 2. Of the 16 papers, 8 used latent class analysis, 2 used latent profile analysis, and 6 used clustering methods. The 16 papers included a total of 15 models (in 14 papers) for which clear clusters/classes were presented. Of these, $9(60.0 \%)$ included 4 classes/clusters; 3 included fewer and 3 included more. For all but one paper, all variables used to form the classes/clusters were based in social power. This included social position variables, like age or gender, processes such as discrimination, and identity-related measures like race centrality. The remaining study created profiles based on diagnoses and medication usage, with subsequent analyses using the resulting profiles stratified by intersections of ethnicity/race and gender. 
Fig. 1 Flow diagram of systematic review process and exclusion criteria. $L C A$ latent class analysis; $L P A$ latent position analysis

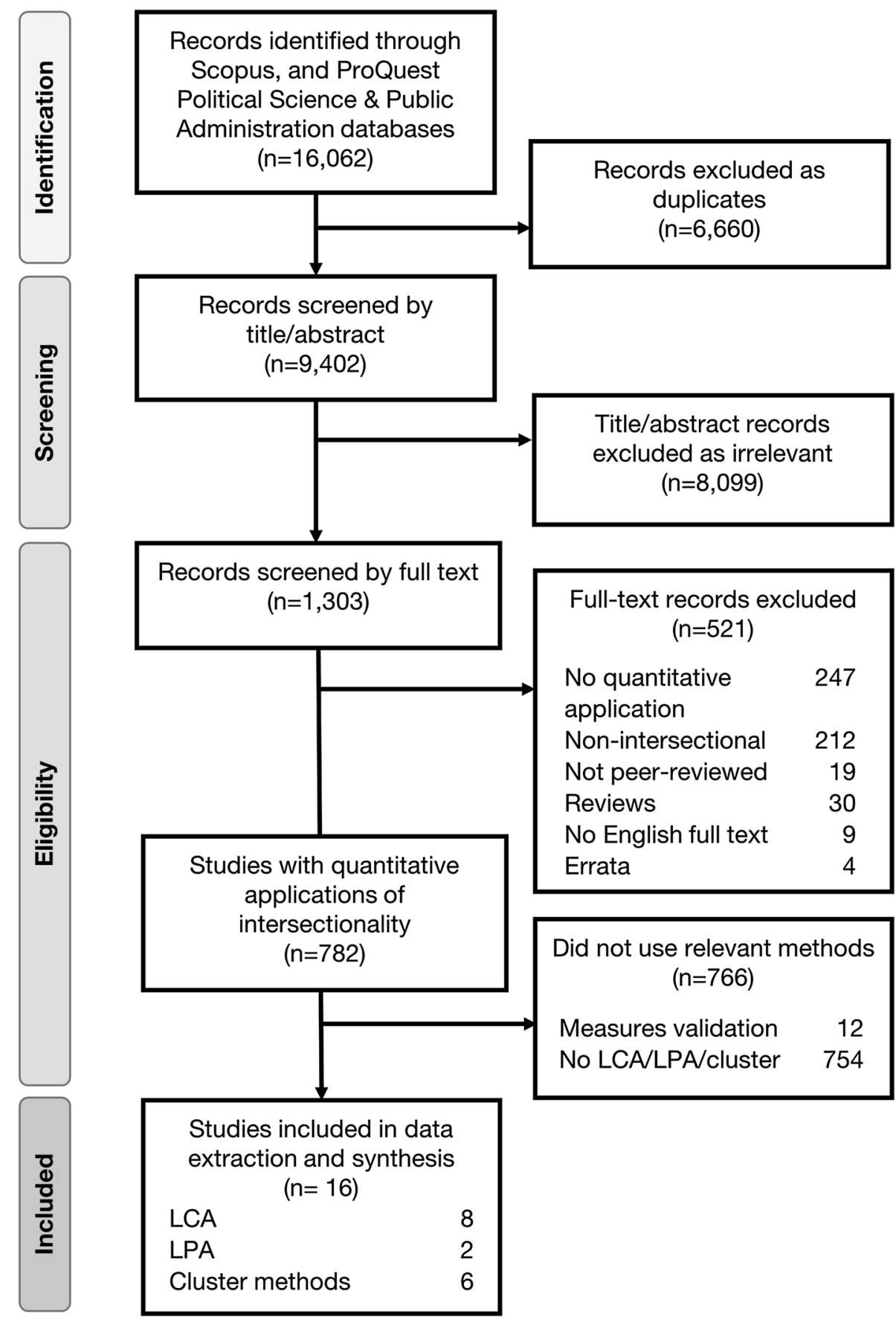

Across studies, the resulting classes/clusters were then frequently used as a variable in additional analyses. Analyses generally either described differences in sociodemographic characteristics between the classes/clusters, or used class/ cluster membership as exposure groups in analyses such as regression analysis, structural equation modeling, mediation, and survival analysis, to predict outcomes. These methods typically resulted in coefficient estimates and statistical inferences regarding the significance of class/cluster membership towards the outcome. Some studies in addition to using clusters as terms in their statistical models, presented overall mean or prevalence estimates for each cluster [31, 32], but not all did. One study performing a cluster analysis presented results visually in a dendrogram, with no specific definition of the final clusters, and no assessment of the clusters relative to any outcome or sociodemographic [33].

Author quotes regarding their methods rationale included both theoretical/intersectional and statistical arguments. Theoretical reasons included the importance of a "person-centered approach", to examine the complexity 
Table 1 Characteristics and indicators of engagement with intersectionality in quantitative intersectionality papers using latent variable or clustering analysis

\begin{tabular}{|c|c|c|}
\hline & \multicolumn{2}{|c|}{$\begin{array}{l}\text { Total } \\
(n=16)\end{array}$} \\
\hline & $n$ & $\%$ \\
\hline Health-related outcome & 10 & 62.5 \\
\hline \multicolumn{3}{|l|}{ Journal discipline $^{\mathrm{a}}$} \\
\hline Psychology & 9 & 56.3 \\
\hline Other social sciences ${ }^{b}$ & 4 & 25.0 \\
\hline Children and youth & 4 & 25.0 \\
\hline Medical and life science & 3 & 18.8 \\
\hline Sociology & 2 & 12.5 \\
\hline Population/public health and safety & 2 & 12.5 \\
\hline Education & 2 & 12.5 \\
\hline Other sciences ${ }^{\mathrm{c}}$ & 1 & 6.3 \\
\hline \multicolumn{3}{|l|}{ Country of first author } \\
\hline United States & 11 & 68.8 \\
\hline United Kingdom & 4 & 25.0 \\
\hline Germany & 1 & 6.3 \\
\hline \multicolumn{3}{|l|}{ Study type } \\
\hline Quantitative & 14 & 87.5 \\
\hline Mixed-methods & 2 & 12.5 \\
\hline \multicolumn{3}{|l|}{ Study design } \\
\hline Cross-sectional study & 16 & 100.0 \\
\hline Complex multi-stage sample & 4 & 25.0 \\
\hline $\begin{array}{l}\text { Data from census or population records (e.g., } \\
\text { birth records) }\end{array}$ & 0 & 0.0 \\
\hline Intersectionality defined & 14 & 87.5 \\
\hline Cited foundational author(s) & 15 & 93.8 \\
\hline \multicolumn{3}{|l|}{ Engagement with methodology papers ${ }^{\mathrm{a}}$} \\
\hline 0 cited & 4 & 25.0 \\
\hline 1 cited & 7 & 43.8 \\
\hline $2-4$ cited & 4 & 25.0 \\
\hline $5+$ cited & 1 & 6.3 \\
\hline
\end{tabular}

${ }^{a}$ Multiple disciplines per journal; proportions do not sum to $100 \%$

${ }^{\mathrm{b}}$ Other than psychology, sociology, criminology

${ }^{\mathrm{c}}$ Other than medical and life sciences, or physical, earth or space sciences

${ }^{\mathrm{d}}$ Based on the list of 45 methodology papers included in a larger review [1]

of co-occurring experiences, to identify intersectional subgroups or response patterns, to find intersectional constructs such as intersectional stigma or discrimination, and appropriateness to researchers' relative power in data analysis. One study using multidimensional scaling analysis describing the method as a way of applying an "idiographic" approach, which is an approach that reveals underlying or hidden constructs [34]. Author rationales are summarized in Table 3, and illustrated with sample quotes.
Statistical arguments sometimes focused on the advantage of methods such as LCA over non-clustering methods. LCA was seen as useful in that it was a data-driven exploratory approach [35], and that it did not require a priori specification of groups [36]. The groups were noted to be potentially both more homogenous [37] and substantively meaningful $[35,38]$, especially useful when groups are then used as categorizations to predict outcomes. For example, one study stated, "Using an LCA approach allowed us to define more cohesive social groups and subsequently the reference group in the regression analyses was also likely to be a more homogenous group, which increases the validity of the analyses." [37]. Some authors provided a rationale for choosing one type of latent variable or clustering method over others. Cited advantages of LCA over other clustering methods included the availability of model fit statistics to aid in decision-making regarding class number $[35,36]$. In practice, all LCA/LPA studies in our review used fit statistics, and some also considered theory or interpretability of the final classes [31, 32, 37-40]. Additionally, certain cluster studies utilized statistical criteria as well, either alone $[30,38])$ ) or in combination with interpretability [43]. Other stated advantages of LCA compared to cluster methods included the model-based nature of the method allowing for groupings to be tested in independent samples to confirm generalizability [36], and analyses allowing for bootstrapping estimations [35].

In using LCA to combine multiple indicators of socioeconomic status, it was noted to better show nuanced differences than methods that combine socioeconomic indicators into a single continuous variable (e.g., principal components analysis) [37]. In contrast, authors using hierarchical clustering or other non-LCA/LPA methods cited advantages such as not assuming independence of observed variables, and the ability to accommodate both continuous and categorical variables [43].

\section{Discussion}

Included studies were those published in English. Given intersectionality's roots in the United States, relatively few studies have been published in other languages, but those are not represented here. While intersectionality approaches have been used in quantitative research across a wide range of disciplines [1], latent variable and clustering methods in the current study were used primarily in psychology, social sciences, and health research. This reflects the roots of latent variable methods in mental health research, and disciplinary culture regarding methods. In comparison with our larger systematic review [1], authors using these methods seemed more engaged with intersectionality, as evidenced by the provision of definitions ( $87.5 \%$ vs. $73.1 \%$ in the larger literature), citation of foundational sources ( $93.8 \%$ vs. $68.0 \%)$ 
226

Social Psychiatry and Psychiatric Epidemiology (2022) 57:221-237
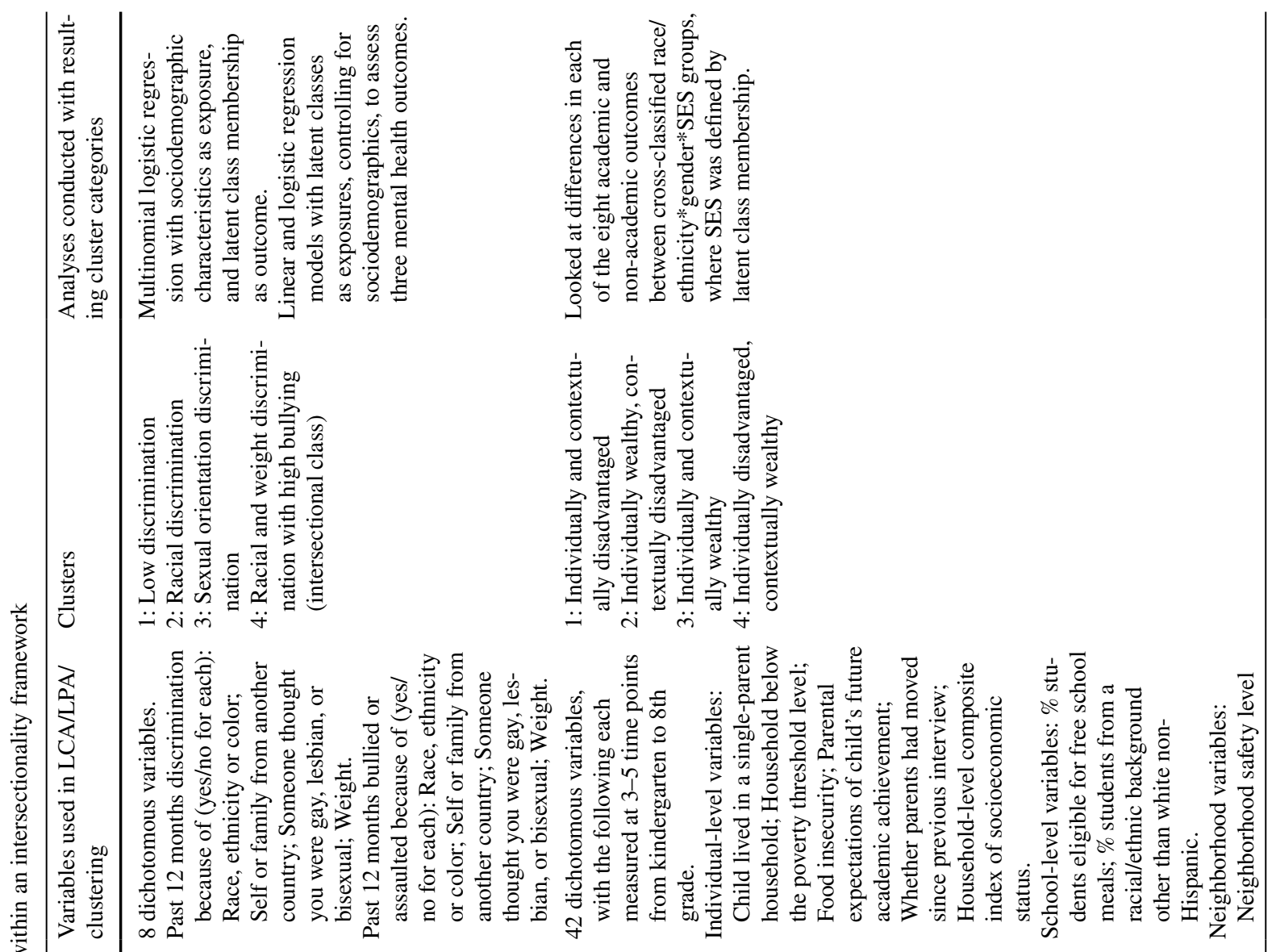

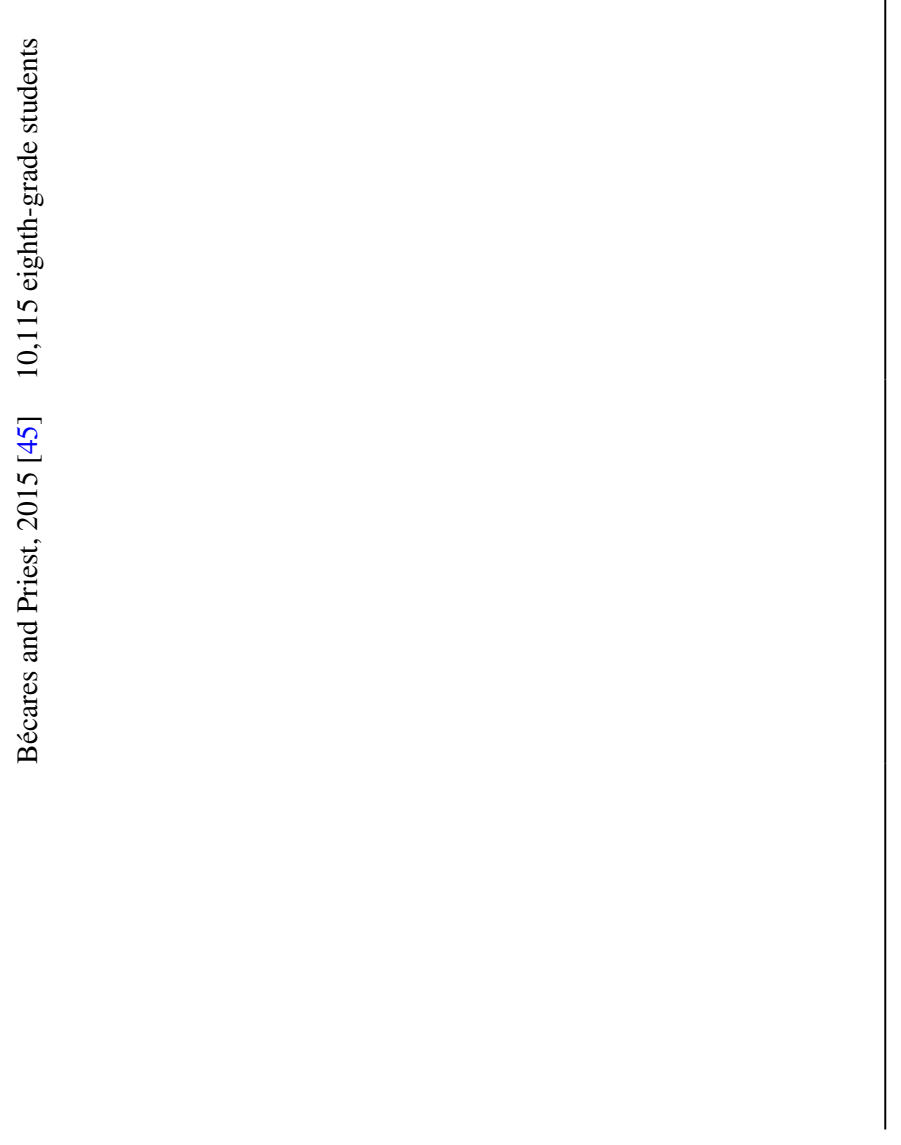

Springer 


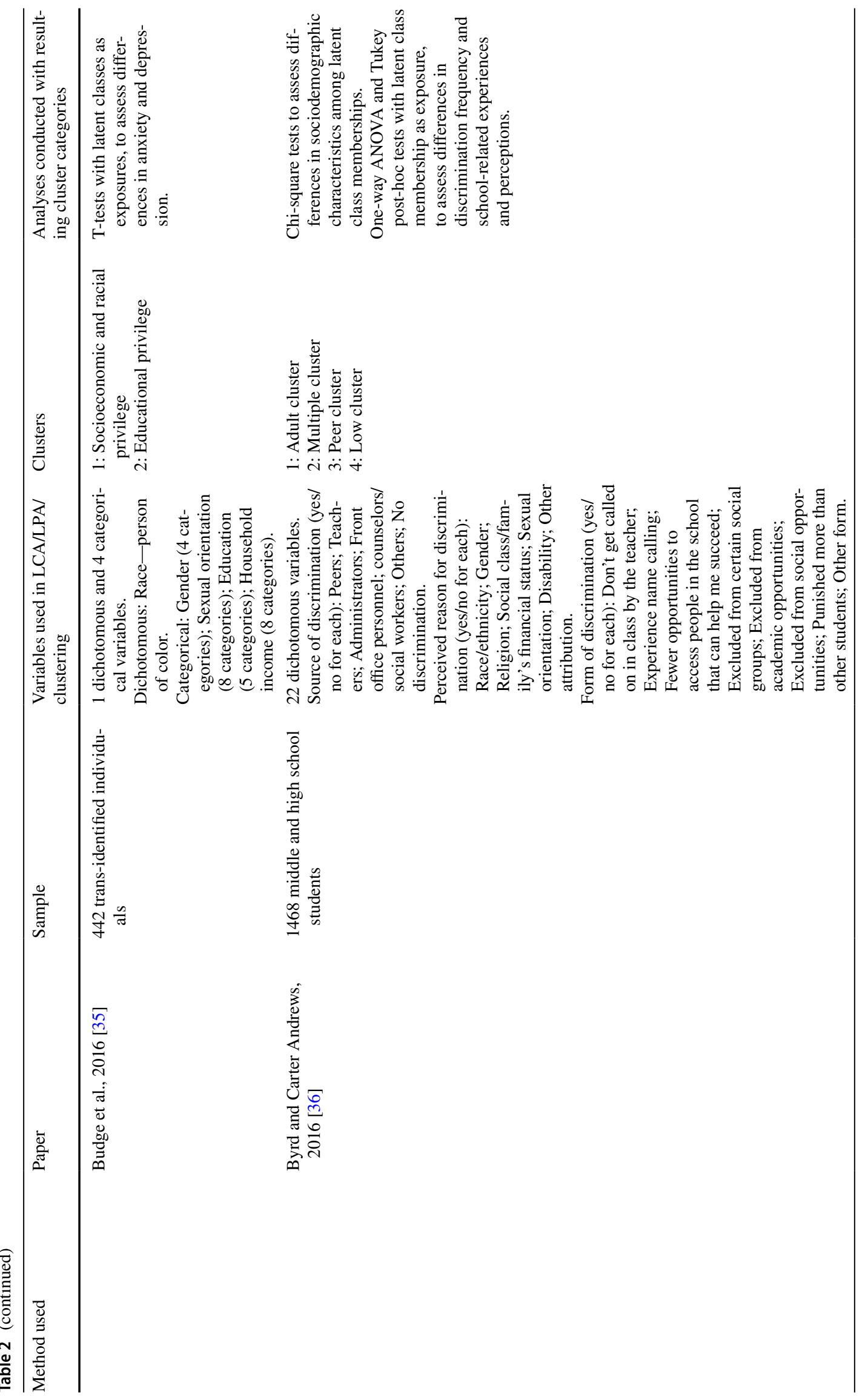




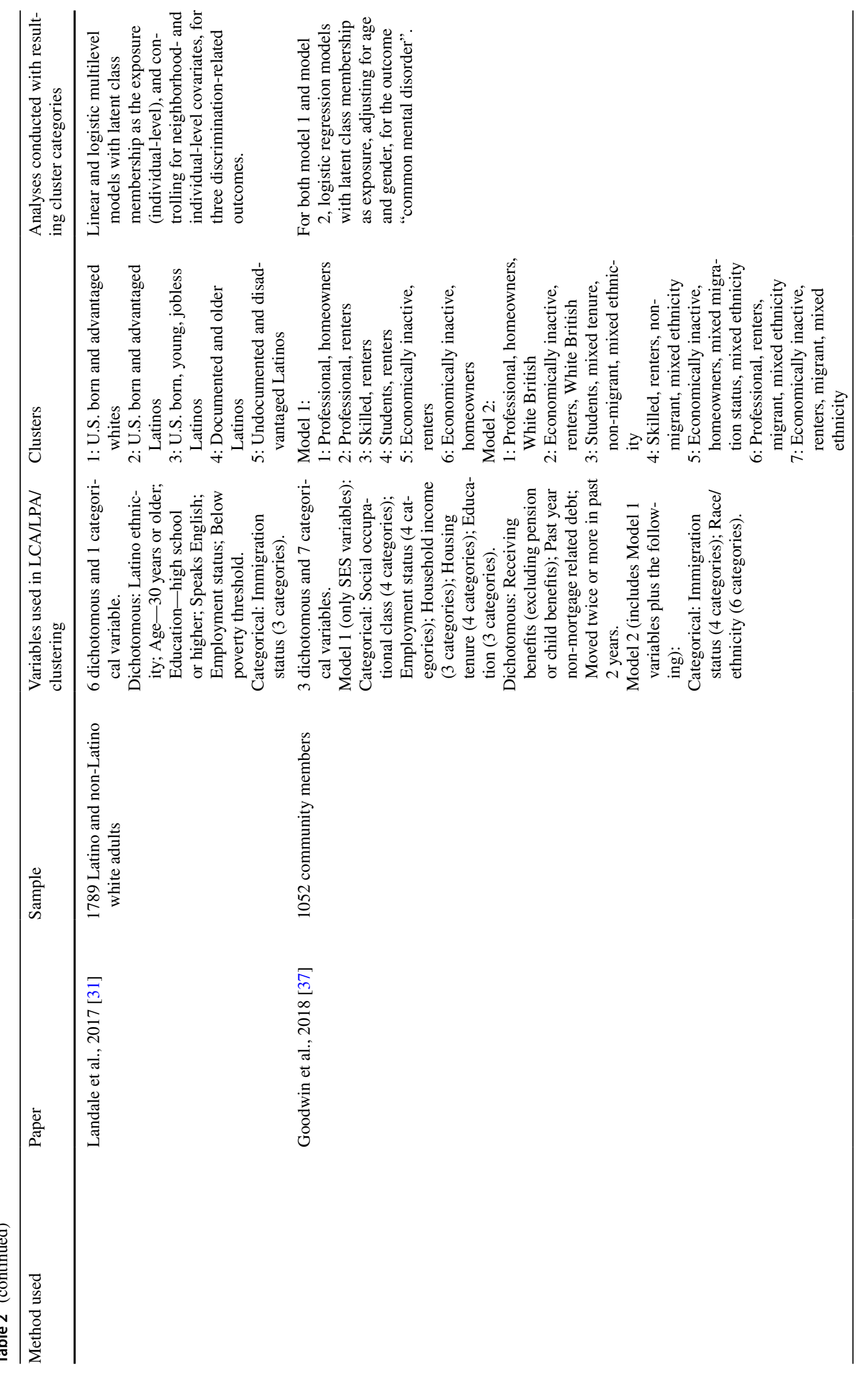




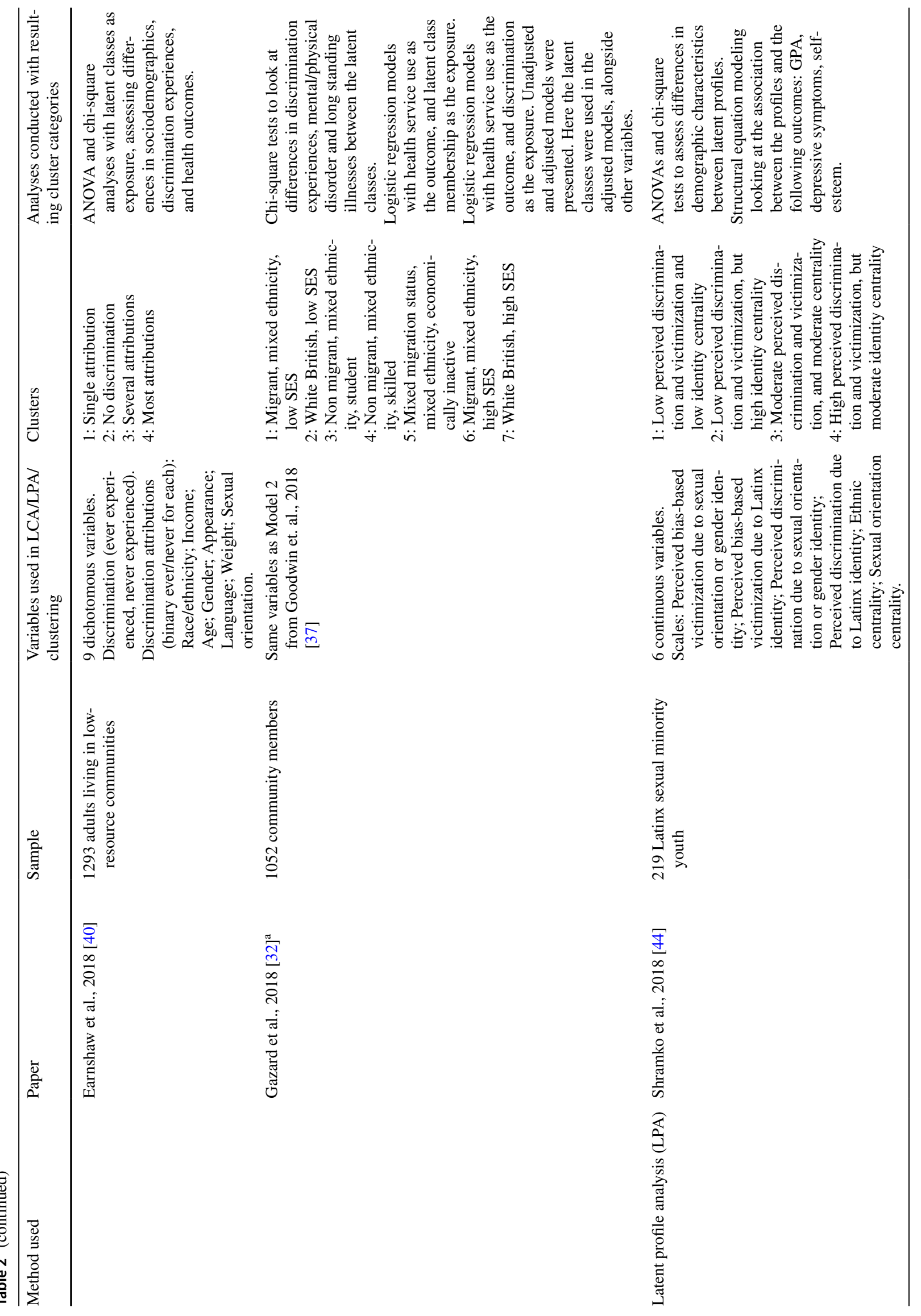




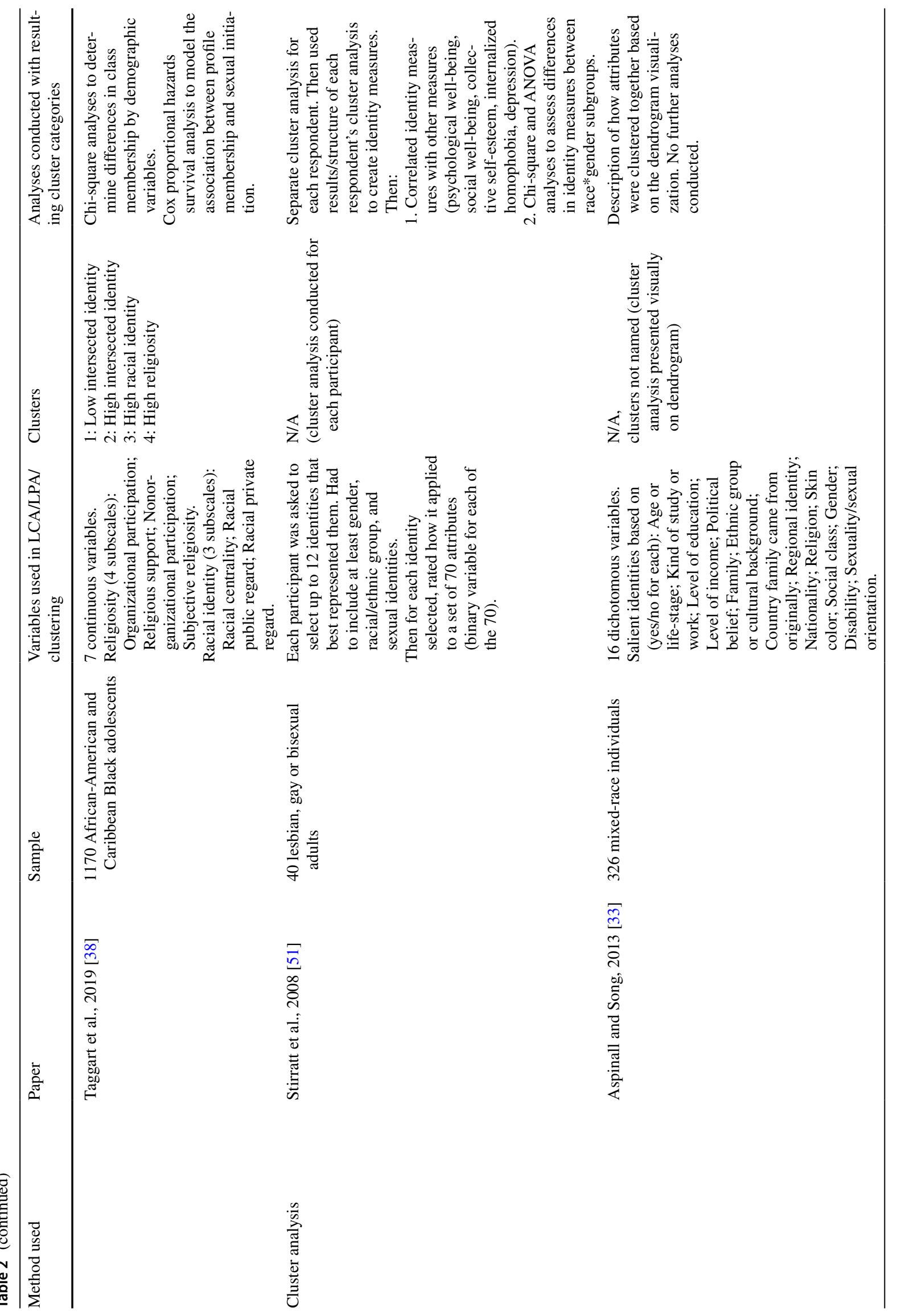




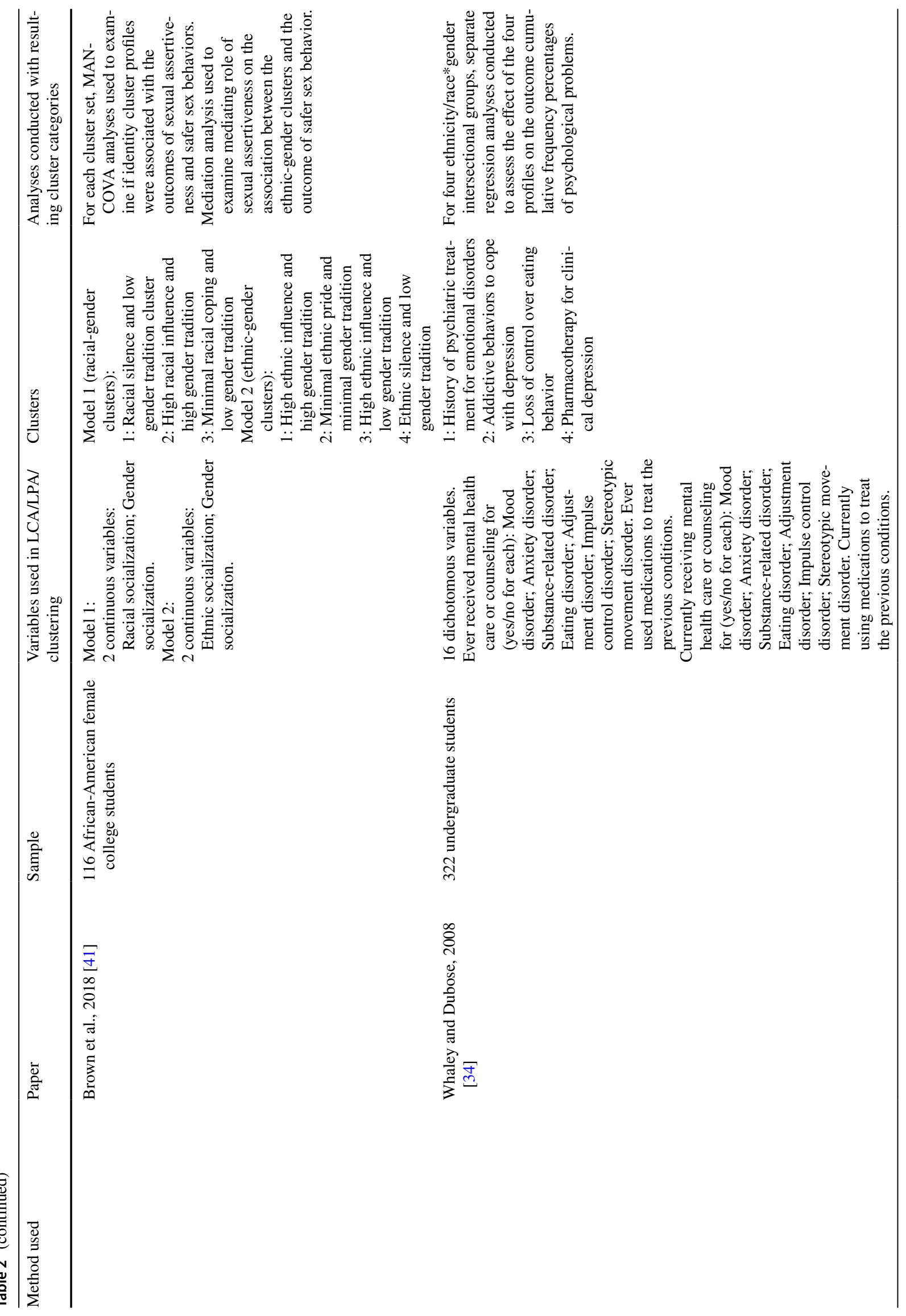




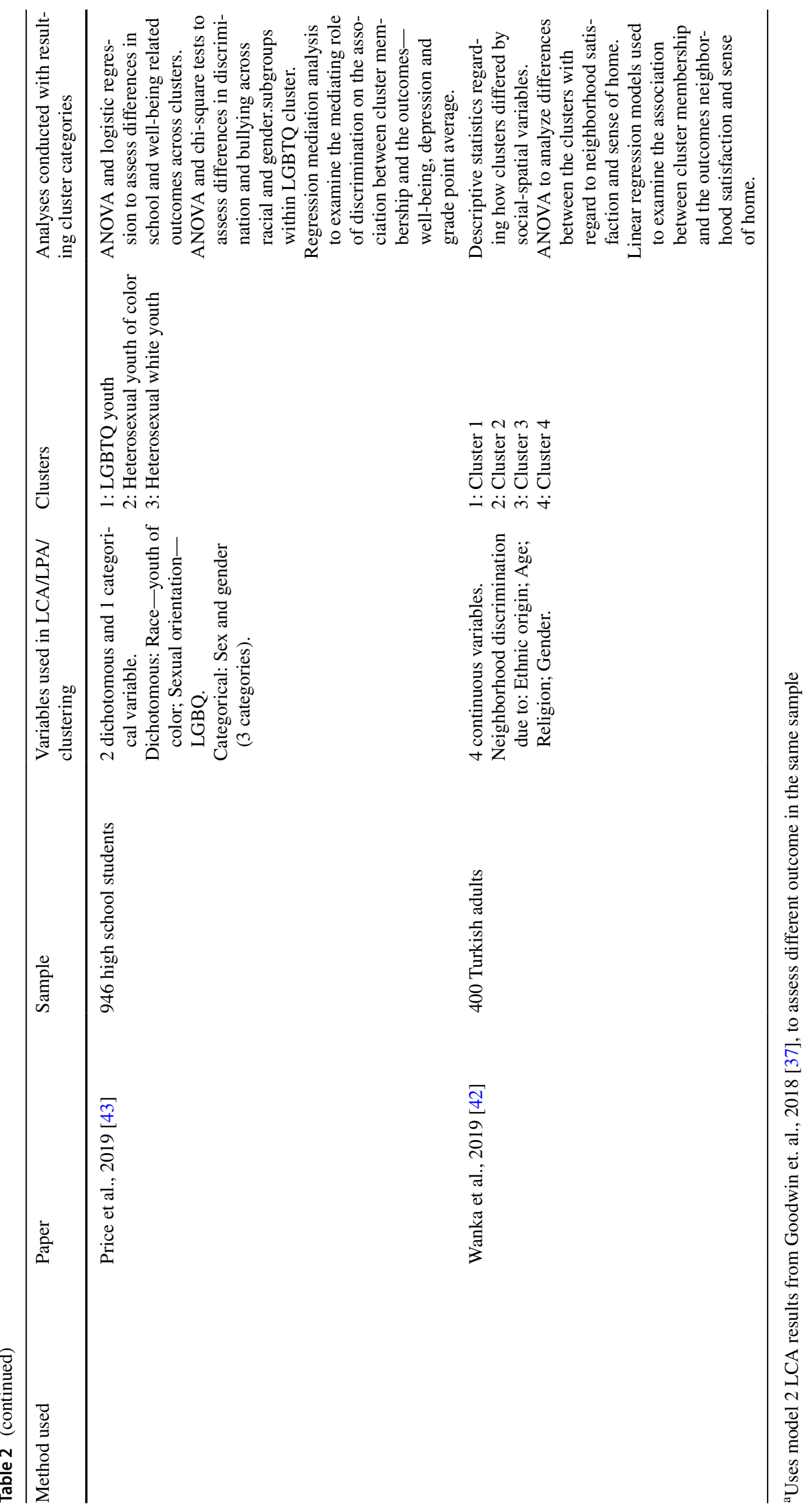


Table 3 Theoretical rationale provided by authors for use of latent variable or clustering methods within an intersectionality framework

\begin{tabular}{|c|c|}
\hline Rationale & Exemplar quotes \\
\hline Person-centered approach & $\begin{array}{l}\text { "The intersectionality framework proposes that individual experiences must be } \\
\text { examined in the context of simultaneous memberships in different status groups. } \\
\text { In emphasizing constellations of multiple statuses, this framework requires a } \\
\text { person-oriented approach instead of a variables-oriented approach" [31] }\end{array}$ \\
\hline Examining the complexity of co-occurring experiences & $\begin{array}{l}\text { "Discrimination and bullying are complex social phenomena ... As opposed to } \\
\text { attempting to disentangle the different attributes of discrimination and bullying, } \\
\text { LCA can examine how they jointly co-occur" [39] } \\
\text { "LCA can create a series of classes that allows for the study of not only multiple } \\
\text { disadvantaged positions, but also those positions of privilege, as well as positions } \\
\text { that occupy both" [37] }\end{array}$ \\
\hline Identifying intersectional subgroups or response patterns & $\begin{array}{l}\text { "With trans populations often treated as one homogeneous group, it is unclear } \\
\text { how levels of depression and anxiety may differ across trans subgroups or for } \\
\text { individuals holding other minority identities. Therefore, we used cluster analysis } \\
\text { to identify patterns across social identities". [35] } \\
\text { "In this investigation, we were attempting to determine which patterns (i.e. the } \\
\text { quality) of racial-gender and ethnic-gender socialization were reported by partici- } \\
\text { pants." [41] }\end{array}$ \\
\hline Finding intersectionality & $\begin{array}{l}\text { "We first employed latent class analysis to understand who reports intersectional } \\
\text { experiences of discrimination." [40] } \\
\text { "Idiographic and idiothetic approaches using MDS [multidimensional scaling } \\
\text { analyses] provided evidence of intersectionality for African-American college } \\
\text { students." [34] }\end{array}$ \\
\hline Appropriate to researchers' relative power and privilege & $\begin{array}{l}\text { "[C]luster analysis ... is appropriate given the lack of existing empirical evidence } \\
\text { to test more explicit associations of study constructs. ... helps us to minimize the } \\
\text { assumptions we may make as researchers with relative power and privilege in } \\
\text { absence of guiding theory and empirical support for testing more specific associa- } \\
\text { tions between study constructs." [35] }\end{array}$ \\
\hline
\end{tabular}

and citation of any of a list of key intersectionality methods papers $(75.0 \%$ vs. $53.0 \%)$. Below, we highlight considerations regarding the match between applications of latent variable and clustering methods and intersectional tenets and research aims, address some limitations of current approaches, and highlight missed opportunities for additional uses of these methods.

\section{"Person-centered" approaches and relationality}

Authors commonly described both latent variable and clustering methods as person-centered rather than variable-centered methods [31, 36, 38, 43-45]. This distinction emerged in personality psychology in the 1970s as a way to understand individuals based on multiple personalityrelated variables that were seen to pattern in specific ways within individuals [46]. In research applications, personcentered approaches came to be understood as a search for groups of individuals who were homogenous with regard to whether and how particular variables affected others [11, 47, 48]. In epidemiological terms, person-centeredness would then appear to be about using multiple measures to identify meaningful categories of individuals across which there is causal effect modification for associations under study. However, classes/clusters were not used in this way in any of the included studies, and understandings of person-centeredness in published papers were broader or unarticulated.

It is easy to see how the language of person-centeredness may be appealing to researchers taking an intersectional approach, in that it may be seen as mapping onto the core intersectionality concept of relationality. Relationality is the idea that phenomena such as race/ethnicity, sex/gender and social class are interconnected and maintained through relational processes [24]. Thus, health at an intersection, for example, cannot be understood as the sum of its social identity parts [7]. A relevant aspect of relationality here is co-formation, in which old categories may not be useful and new co-formed ones may be needed [24]. While not labeled clearly as such, this concept appeared to be what some authors referenced when they wrote about identifying an "intersectional class" [39] or finding "evidence of intersectionality" [34]. As intersectionality is more commonly understood in research as an analytic approach or paradigm than a finding [23, 49], it may be more appropriate to be specific regarding findings, for example describing finding evidence to support co-formation of gender and race rather than finding intersectionality itself. 


\section{Intersectional stigma versus intersections of social identities or positions}

While an occasional study brought social intersections into the analysis after clustering (e.g., analyzed effects of intersection on outcome within strata of mental health clusters [34]), most analyses conceptualized intersectionality as primarily represented within the classes/clusters. These approaches comprised two groups based on objectives for implementing latent variable or clustering methods, the first creating intersectional classes/clusters reflecting stigma or social processes, and the second reflecting classes/clusters of social identities/positions.

Many papers in the first group of studies sought to create classes/clusters based on stigma, discrimination, or victimization in ways that traversed different social positions (e.g., by combining measures such as homophobia and racism) [36, 39, 40, 42, 44]. This use harkens back to Berger's foundational work on intersectional stigma, wherein she notes it is composed of influences that can be tied to specific social positions, but which in totality create qualitatively different forms of intersectional stigma [10]. Other authors took a process-based approach focused on socialization, community involvement, or identity centrality, rather than discrimination $[38,41]$. There were two general approaches to these studies of intersectional processes. In the first, researchers analyzed processes within a particular intersection, such as African-American women [41] or sexual minority Latinx youth [44]. In the second, they aimed to create process-based classes in general samples, such as students [39] or residents of a low-resource geographic area [40]. The first approach seems to clearly map onto an intracategorical approach to intersectional complexity, which focuses on specific experiences within an intersection [50], while the second may capture intercategorical categories of stigma or other social processes.

A second group of studies sought to create classes based on social identities or positions themselves, using three main approaches. The first was to create a set of classes/clusters based on a standard set of social positions, such as race, gender, income, and immigration status [32, 35, 37]. The second was to use these methods to create a single measure of a multidimensional construct, for example socioeconomic status $[37,45]$; these classes could then be combined with other social positions to form intersections. The classes/clusters created were then typically used as a variable in additional analyses. Another identity application focused specifically on within-person interaction of identities, rather than their relationship with other variables. This involved more unconventional approaches, such as performing a separate cluster analysis for each person [51] or assessing the correlation between identities for each person in a matrix, then using that matrix data to do the cluster analysis [33].

While the creation of classes/clusters from a standard set of social identities/positions to create "intersections" was common in the included studies, the limitations of this application were generally left unacknowledged with regard to both statistical assumptions and to applicability and interpretability of results to those living at different social intersections. Statistically, the assumption of conditional independence in LCA would require that latent class membership explain all the shared variance between included social identities/positions (e.g., income, race, trans identity, sexual orientation, and education [35]). This could require that entrenched disparities (e.g., racial disparities in income), be fully explained by latent "intersections", a substantial assumption. We note that Bayesian LCA may be useful in detecting and addressing violations of conditional independence [14]. With regard to interpretability, we acknowledge the appeal of using cluster methods in this way to incorporate multiple social position variables into analyses with smaller sample sizes. For example, it is more feasible to condense six binary social position variables into one four-category variable, rather than conduct an analysis of $64\left(2^{6}\right)$ intersectional categories. However, presenting class/cluster results as "intersections" may mask important heterogeneity and limit interpretability. Classes/ clusters based on social identities/positions are not homogenous in membership. LCA classes are based in conditional probabilities, and the resulting classes may not correspond to identifiable groups for health-related interventions. This suggests a trade-off between simplified data and potential applicability to real-world intersections.

\section{Data-driven versus theory-driven approaches}

The tension between theory-driven and data-driven methods in data analysis was clear in the papers' discussions of advantages and disadvantages. Data-driven approaches do allow for condensing a large number of variables in the absence of a theoretical basis for classification. Some researchers stated that the more data-driven nature of these methods serves to mitigate researchers' relative power by reducing the impact of researchers' assumptions [35]. Nevertheless, theory and subjectivity were still required both in the choice of variables used and in the naming and interpretation of resulting classes/clusters. Moreover, researchers often do have information on which to base a choice of intersection for study, and a wealth of qualitative and quantitative research, and community knowledge, to draw from in making these decisions. Moreover, deliberate incorporation of community knowledge can be structured into research team 
membership and processes, as well as study design, addressing issues of social power that are central to intersectionality $[23,52]$. As such, while particular steps in the approaches used may be described as data-driven, in truth all papers we included drew on theory as well. We note that if there is a strong argument for data-driven analytic approaches, it would also apply to other methods (e.g., decision trees [1]) that also serve to take a data-driven approach to reducing intersectional categories in high-dimensional data [53]. Unlike latent variable or cluster methods, decision tree methods result in clearly defined categories by identity/position.

\section{Advantages and further opportunities for latent variable and clustering methods}

The ability of latent variable and clustering methods to allow for overlapping and co-occurring experiences or identities/ positions (and to statistically accommodate correlated variables) allows this set of methods to play a unique role in moving complex theory into quantitative analysis. Under what conditions are these methods most advantageous? While latent variable or clustering methods can result in categories that are similar to those that would have been logically coded, they also have the potential to identify multidimensional constructs that may represent qualitatively different categories of experience (e.g., individual and contextual wealth and disadvantage throughout childhood [45]). This reflects the original aspirations of person-centeredness. These meaningful new categories can then be incorporated into more conventional analyses.

Combining continuous variables is also a strength of latent position and clustering methods. While a series of categorical variables may be cross-coded into categories (potentially representing intersections), cross-classifying continuous variable would first require categorizing each variable. Given that cut-points are often arbitrary and categorization results in loss of information, latent profile analysis or clustering have an advantage in retaining full information, and can be used to create a categorical profile variable that may be more meaningful.

Across the reviewed papers, we noted potential uses of latent variable and clustering methods that were not observed, but which may provide opportunities to contribute to intersectional understandings of health. These missed opportunities included uses in longitudinal analysis, multilevel analysis, effect-measure modification, mediation analysis, and mixed methods. All papers reviewed used cross-sectional analyses, though one coded classes based on multiple retrospective time points. Because co-formation of intersectional experience occurs in social context and over the life course, there is an opportunity to expand analysis over longitudinal periods. While latent variable and clustering methods involve combining measures at a point in time, the resulting classes/clusters represent new categorical variables that could then be used in conjunction with followup data to study how class/cluster membership is associated with later outcomes. Moreover, opportunities to examine how membership in classes itself changes over time (e.g., latent transition analysis, longitudinal LCA) could be taken up in future work.

Quantitative intersectionality researchers have called for greater use of multilevel data to reflect social context and structural factors $[54,55]$. As "person-centered methods" the methods studied were unsurprisingly all applied at the level of the individual. It is worth noting that person-centered methods consider the person in a developmental context and as an "integrated psychological, biological and social being" who must be understood in the context of their environment [48]. While the methods in our review were not designed for nested data, it would also be possible, for example, to conduct an LCA analysis at the group level. We found two attempts to incorporate contextual factors in analyses within this review. One study used factors from three social-ecological levels - the individual, school, and neighborhood-in their LCA analysis, though these were applied to individual-level observations rather than in nested data to create a measure of advantage/disadvantage for each participant [45]. Another study used individual-level variables in an LCA analysis but then used the resulting classes as an individuallevel variable within a multilevel regression [31].

In our review, intersectional classes/clusters were frequently treated as exposure variables in subsequent analyses. We did not note any use as effect-measure modifiers. Given the original conceptualization of person-centered methods as producing homogeneous classes with regard to the effects of exposures on outcomes, and the fit of this conceptualization with intersectionality perspectives that acknowledge differential impacts across intersections, we would recommend further exploration of uses of classes/ clusters as effect-measure modifiers or stratification variables. The potential roles of intersectional stigma classes, for example, could plausibly modify the effects of a range of exposures on health. Such an approach could also be embedded in a mediation analysis that accommodates interaction between cross-coded intersections as an exposure and a variable such as intersectional stigma as a mediator. Intersectional mediation approaches allow for decomposition of the indirect effect into components representing effects of different mediating classes, versus class membership having different effects on the outcome for those at different intersections (mediated interaction) $[26,56]$. Finally, we note that while our review was of quantitative methods, two papers reviewed included mixed methods, which may have additional advantages with regard to an intersectional approach. 


\section{Conclusion}

Researchers applied latent variable or clustering methods in a range of ways that reflected different matches between intersectionality and their data analysis methods. While limitations were noted, there appears to be underdeveloped potential in applying these and related clustering methods to questions of intersectional co-formation, particularly with regard to experiences of stigma, discrimination, and violence, or in identifying and encoding multidimensional constructs representing complex social positioning. The use of these classes/clusters in further analyses would benefit from greater complexity, including use in longitudinal and multilevel studies, and in studies of effect-measure modification. Finally, research would benefit from greater specificity in reporting of rationale for methods and interpretation of findings, to better support the ultimate goal of improving health equity.

Acknowledgements This analysis was funded by the Canadian Institutes of Health Research through a Sex and Gender Science Chair (GSB-171372). The authors would like to thank the following people for work on the larger systematic search and extraction upon which this review is based: Ruo Su Zhang, Alma Villa-Rueda, Sahana Kukan, Fatima Kudaeva, Rachel Girimonte, Siobhan Churchill, and Isabella Aversa.

\section{Declarations}

Conflict of interest On behalf of all authors, the corresponding author states that there is no conflict of interest.

Open Access This article is licensed under a Creative Commons Attribution 4.0 International License, which permits use, sharing, adaptation, distribution and reproduction in any medium or format, as long as you give appropriate credit to the original author(s) and the source, provide a link to the Creative Commons licence, and indicate if changes were made. The images or other third party material in this article are included in the article's Creative Commons licence, unless indicated otherwise in a credit line to the material. If material is not included in the article's Creative Commons licence and your intended use is not permitted by statutory regulation or exceeds the permitted use, you will need to obtain permission directly from the copyright holder. To view a copy of this licence, visit http://creativecommons.org/licenses/by/4.0/.

\section{References}

1. Bauer GR, Churchill SM, Mahendran M et al (2021) Intersectionality in quantitative research: a systematic review of its emergence and applications of theory and methods. SSM Popul Health 14:100798. https://doi.org/10.1016/j.ssmph.2021.100798

2. Combahee River Collective (1977) Combahee River Collective Statement. Boston, MA

3. Collins PH, Bilge S (2020) Getting the history of intersectionality straight? Intersectionality, 2nd edn. Polity Press, Cambridge, pp $72-100$

4. Crenshaw K (1991) Mapping the margins: intersectionality, identity politics, and violence against women of color. Stanford Law $\operatorname{Rev}$ 43:61
5. Crenshaw K (1989) Demarginalizing the intersection of race and sex: a Black feminist critique of antidiscrimination doctrine, feminist theory and antiracist politics. U Chi Legal F 1989:139-168

6. Collins PH (1990) Black feminist thought: knowledge, consciousness, and the politics of empowerment. Taylor \& Francis Group, Florence

7. Bowleg L (2008) When Black + lesbian + woman $\neq$ Black lesbian woman: the methodological challenges of qualitative and quantitative intersectionality research. Sex Roles 59:312-325. https://doi.org/10.1007/s11199-008-9400-z

8. Bowleg L (2013) “Once you've blended the cake, you can't take the parts back to the main ingredients": black gay and bisexual men's descriptions and experiences of intersectionality. Sex Roles 68:754-767. https://doi.org/10.1007/s11199-012-0152-4

9. Turan JM, Elafros MA, Logie CH et al (2019) Challenges and opportunities in examining and addressing intersectional stigma and health. BMC Med 17:7. https://doi.org/10.1186/ s12916-018-1246-9

10. Berger MT (2004) Workable sisterhood: the political journey of stigmatized women with HIV/AIDS. Princeton University Press

11. Muthen B, Muthen LK (2000) Integrating person-centered and variable-centered analyses: growth mixture modeling with latent trajectory classes. Alcohol Clin Exp Res 24:882-891. https://doi.org/10.1111/j.1530-0277.2000.tb02070.x

12. Gensler S (2017) Finite mixture models. In: Homburg C, Klarmann M, Vomberg A (eds) Handbook of Market Research. Springer International Publishing, Cham, pp 1-14

13. Collins LM, Lanza ST (2009) Latent class and latent transition analysis: with applications in the social, behavioral, and health sciences I Wiley. John Wiley and Sons Inc., Hoboken, NJ

14. Lee J, Jung K, Park J (2020) Detecting conditional dependence using flexible Bayesian latent class analysis. Front Psychol. https://doi.org/10.3389/fpsyg.2020.01987

15. Akaike H (1974) A new look at the statistical model identification. IEEE Trans Autom Control 19:716-723. https://doi.org/ 10.1109/TAC.1974.1100705

16. Schwarz G (1978) Estimating the dimension of a model. Ann Stat 6:461-464. https://doi.org/10.1214/aos/1176344136

17. Sclove SL (1987) Application of model-selection criteria to some problems in multivariate analysis. Psychometrika 52:333343. https://doi.org/10.1007/BF02294360

18. Celeux G, Soromenho G (1996) An entropy criterion for assessing the number of clusters in a mixture model. J Classif 13:195212. https://doi.org/10.1007/BF01246098

19. Schreiber JB (2017) Latent class analysis: an example for reporting results. Res Social Adm Pharm 13:1196-1201. https:// doi.org/10.1016/j.sapharm.2016.11.011

20. Nylund-Gibson K, Choi AY (2018) Ten frequently asked questions about latent class analysis. Transl Issues Psychol Sci 4:440-461. https://doi.org/10.1037/tps0000176

21. Li Vigni M, Durante C, Cocchi M (2013) Exploratory data analysis. Data Handling in Science and Technology. Elsevier, pp 55-126

22. Xu R, Wunsch DC (2010) Clustering algorithms in biomedical research: a review. IEEE Rev Biomed Eng 3:120-154. https:// doi.org/10.1109/RBME.2010.2083647

23. Bowleg L (2012) The problem with the phrase women and minorities: intersectionality - an important theoretical framework for public health. Am J Public Health 102:1267-1273. https://doi.org/10.2105/AJPH.2012.300750

24. Collins PH (2019) Relationality within intersectionality. In: Collins PH (eds) Intersectionality as critical social theory. Duke University Press, pp 225-252

25. Shokoohi M, Bauer GR, Kaida A et al (2019) Patterns of social determinants of health associated with drug use among women 
living with HIV in Canada: a latent class analysis. Addiction 114:1214-1224. https://doi.org/10.1111/add.14566

26. Bauer GR, Scheim AI (2019) Methods for analytic intercategorical intersectionality in quantitative research: discrimination as a mediator of health inequalities. Soc Sci Med 226:236-245. https://doi.org/10.1016/j.socscimed.2018.12.015

27. Moher D, Liberati A, Tetzlaff J et al (2009) Preferred reporting items for systematic reviews and meta-analyses: the PRISMA statement. BMJ 339:b2535-b2535. https://doi.org/10.1136/bmj. b2535

28. Covidence Systematic Review Software [computer software]. Veritas Health Innovation, Melbourne, AUS. https://www.covid ence.org/

29. (2020) Ulrichs Serials Analysis System (USAS). https://www.ulric hsweb.com/ulrichsweb/analysis/. Accessed 16 Nov 2020

30. SAS [computer software]. SAS Institute, Cary, NC. https://www. sas.com/en_us/home.html

31. Landale NS, Oropesa RS, Noah AJ (2017) Experiencing discrimination in Los Angeles: Latinos at the intersection of legal status and socioeconomic status. Soc Sci Res 67:34-48. https://doi.org/ 10.1016/j.ssresearch.2017.05.003

32. Gazard B, Chui Z, Harber-Aschan L et al (2018) Barrier or stressor? The role of discrimination experiences in health service use. BMC Public Health 18:1354. https://doi.org/10.1186/ s12889-018-6267-y

33. Aspinall PJ, Song M (2013) Is race a 'salient...' or 'dominant identity' in the early 21 st century: the evidence of UK survey data on respondents' sense of who they are. Soc Sci Res 42:547-561. https://doi.org/10.1016/j.ssresearch.2012.10.007

34. Whaley AL, Dubose J (2018) Intersectionality of ethnicity/race and gender in the phenomenology of African American college students' presenting problems: a profile analysis using nonmetric multidimensional scaling. Int J Adv Couns 40:279-297. https:// doi.org/10.1007/s10447-018-9326-2

35. Budge SL, Thai JL, Tebbe EA, Howard KAS (2016) The intersection of race, sexual orientation, socioeconomic status, trans identity, and mental health outcomes. Couns Psychol 44:1025-1049. https://doi.org/10.1177/0011000015609046

36. Byrd CM, Carter Andrews DJ (2016) Variations in students' perceived reasons for, sources of, and forms of in-school discrimination: a latent class analysis. J Sch Psychol 57:1-14. https://doi.org/ 10.1016/j.jsp.2016.05.001

37. Goodwin L, Gazard B, Aschan L et al (2018) Taking an intersectional approach to define latent classes of socioeconomic status, ethnicity and migration status for psychiatric epidemiological research. Epidemiol Psychiatr Sci 27:589-600. https://doi.org/ $10.1017 /$ S2045796017000142

38. Taggart T, Powell W, Gottfredson N et al (2019) A person-centered approach to the study of Black adolescent religiosity, racial identity, and sexual initiation. J Res Adolesc 29:402-413. https:// doi.org/10.1111/jora.12445

39. Garnett BR, Masyn KE, Austin SB et al (2014) The Intersectionality of discrimination attributes and bullying among youth: an applied latent class analysis. J Youth Adolesc 43:1225-1239. https://doi.org/10.1007/s10964-013-0073-8

40. Earnshaw VA, Rosenthal L, Gilstad-Hayden K et al (2018) Intersectional experiences of discrimination in a low-resource urban community: an exploratory latent class analysis. J
Community Appl Soc Psychol 28:80-93. https://doi.org/10. 1002/casp. 2342

41. Brown DL, Blackmon S, Shiflett A (2018) Safer sexual practices among African American women: intersectional socialisation and sexual assertiveness. Cult Health Sex 20:673-689. https://doi.org/ 10.1080/13691058.2017.1370132

42. Wanka A, Wiesböck L, Allex B et al (2019) Everyday discrimination in the neighbourhood: what a 'doing' perspective on age and ethnicity can offer. Ageing Soc 39:2133-2158. https://doi.org/10. 1017/S0144686X18000466

43. Price M, Polk W, Hill NE et al (2019) The intersectionality of identity-based victimization in adolescence: a person-centered examination of mental health and academic achievement in a U.S. high school. J Adolesc 76:185-196. https://doi.org/10.1016/j. adolescence.2019.09.002

44. Shramko M, Toomey RB, Anhalt K (2018) Profiles of minority stressors and identity centrality among sexual minority Latinx youth. Am J Orthopsychiatry 88:471-482. https://doi.org/10. 1037/ort0000298

45. Bécares L, Priest N (2015) Understanding the influence of race/ ethnicity, gender, and class on inequalities in academic and nonacademic outcomes among eighth-grade students: findings from an intersectionality approach. PLoS ONE 10:e0141363. https:// doi.org/10.1371/journal.pone.0141363

46. Block J (1971) Lives through time. Bancroft Books, Berkeley

47. Laursen BP, Hoff E (2006) Person-centered and variable-centered approaches to longitudinal data. Merrill-Palmer Q 52:377-389. https://doi.org/10.1353/mpq.2006.0029

48. Magnusson D (2003) The person approach: concepts, measurement models, and research strategy. New Dir Child Adolesc Dev 2003:3-23. https://doi.org/10.1002/cd.79

49. Cho S, Crenshaw KW, McCall L (2013) Toward a field of intersectionality studies: Theory, applications, and praxis. Signs J Women Cult Soc 38:785-810. https://doi.org/10.1086/669608

50. McCall L (2005) The complexity of intersectionality. Signs 30:1771-1800. https://doi.org/10.1086/426800

51. Stirratt MJ, Meyer IH, Ouellette SC, Gara MA (2008) Measuring identity multiplicity and intersectionality: hierarchical classes analysis (HICLAS) of sexual, racial, and gender identities. Self and Identity 7:89-111. https://doi.org/10.1080/152988607012522 03

52. Agénor M (2020) Future directions for incorporating intersectionality into quantitative population health research. Am J Public Health 110:803-806. https://doi.org/10.2105/AJPH.2020.305610

53. Mahendran M, Lizotte DJ, Bauer GR (forthcoming) Describing intersectional health outcomes: an evaluation of quantitative data analysis methods. Epidemiology

54. Bowleg L, Bauer G (2016) Invited reflection: quantifying intersectionality. Psychol Women Q 40:337-341. https://doi.org/10. 1177/0361684316654282

55. Bauer GR (2014) Incorporating intersectionality theory into population health research methodology: challenges and the potential to advance health equity. Soc Sci Med 110:10-17. https://doi.org/ 10.1016/j.socscimed.2014.03.022

56. Jackson JW, VanderWeele TJ (2019) Intersectional decomposition analysis with differential exposure, effects, and construct. Soc Sci Med 226:254-259. https://doi.org/10.1016/j.socscimed.2019.01. 033 